\section{Left lower lobe ventilation and its relation to cardiomegaly and posture}

\author{
M S M Alexander, R N Arnot, J P Lavender
}

Departments of Diagnostic

Radiology and Medical

Physics, Royal

Postgraduate Medical

School, Hammersmith

Hospital, London

W12 0HS

M S M Alexander, FRCR, senior registrar

R N Arnot, FINSTP, senior

physicist

J P Lavender, FRCP, professor

Correspondence to:

Dr Alexander.

BrMed f 1989;299:94
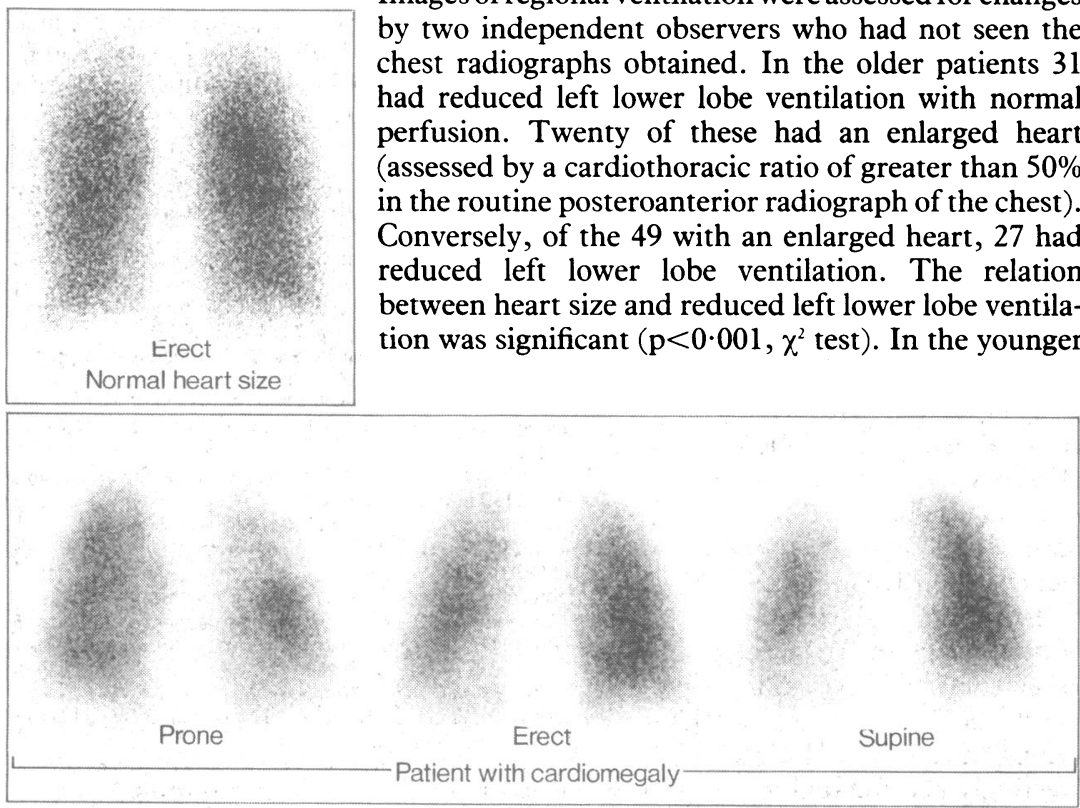

Posterior ventilation images showing normal ventilation and changes of left lower lobe ventilation with position in a patient with cardiomegaly

\section{Subjects, methods, and results}

We compared routine ventilation (krypton-81m) and perfusion (technetium-99m macroaggregate) scans obtained in 90 elderly patients (aged 65 and over) with those obtained in 90 younger patients (aged 20-40). Images of regional ventilation were assessed for changes by two independent observers who had not seen the chest radiographs obtained. In the older patients 31 had reduced left lower lobe ventilation with normal perfusion. Twenty of these had an enlarged heart (assessed by a cardiothoracic ratio of greater than $50 \%$ in the routine posteroanterior radiograph of the chest). Conversely, of the 49 with an enlarged heart, 27 had reduced left lower lobe ventilation. The relation between heart size and reduced left lower lobe ventilation was significant $\left(p<0 \cdot 001, \chi^{2}\right.$ test $)$. In the younger group eight patients had an enlarged heart and three of these showed reduced left lower lobe ventilation. None of those with normal sized hearts had reduced left lower lobe ventilation.

We studied the effect of posture by recording posterior views of ventilation in the supine, erect, and prone positions in a further 19 unselected elderly patients and in the supine and prone positions in 10 normal subjects. Eleven of the elderly patients had enlarged hearts, of whom seven had reduced left lower lobe ventilation in the erect position; the ventilation was improved in the prone position in all seven but was worse in the supine position in five (figure). Four out of the 10 patients with normal ventilation in the erect position had an increased heart size, and a change to the supine position reduced the left lower lobe ventilation in one of these four. Two of the patients with reduced left lower lobe ventilation had a normal heart size, and a change in posture had no effect. No changes in ventilation were observed at the right base. In the normal volunteers no change was recorded when they moved from the prone to the supine position.

\section{Department of \\ Microbiology, York \\ District Hospital, York YO3 7HE \\ Jeremy Haynes, MRCPATH, senior registrar \\ Department of Microbiology, University of Leeds, Leeds LS2 9JT Peter M Hawkey, MRCPATH, senior lecturer}

Correspondence to: $\mathrm{Dr}$ Haynes.

\section{Providencia alcalifaciens and travellers' diarrhoea}

\author{
Jeremy Haynes, Peter M Hawkey
}

The genus Providencia has been implicated as a cause of diarrhoea, particularly in children. ${ }^{1-3}$ We looked for Providencia alcalifaciens in stool specimens from a group of patients with travellers' diarrhoea and a group of patients with diarrhoea but no history of recent foreign travel.

\section{Patients, methods, and results}

From August to October 1987 and July to October 1988 we studied stool samples from patients with

\section{Comment}

Our data suggest that a partial loss of ventilation in the left lower lobe may result from cardiomegaly and that this change is related to posture. Even in the erect position this loss of ventilation is seen in more than half of the routine ventilation perfusion scans of subjects with cardiomegaly; in some the left lower lobe seems not to be ventilated at all. Compression of the major airways by an enlarged left atrium has been shown to occur in infants ${ }^{23}$ but is not evident on computed tomography in adults with cardiomegaly in the supine position. Movement of the diaphragm may be restricted by an enlarged heart, but the most likely cause of the loss of ventilation is compression of the lung and minor airways. This may explain why collapse of the lower lobe in patients after operation is more commonly left sided. After cardiac operations such collapse may occur in up to $60 \%$ of patients. ${ }^{4} \mathrm{~A}$ simple change in position from supine to prone moving the heart away from the left lower lobe may improve ventilation in hypoxic patients with cardiomegaly.

1 MacNalty AS, ed. The British medical dictionary. London: Caxton, 1901:528. 2 Cochran ST, Gyepes MT, Smith LE. Obstruction of the airways by the hear and pulmonary vessels in infants. Pediatr Radiol 1977;6(2):81-7.

3 Rivkin LM, Read RC, Lillehei CW, Varco RC. Massive atelectasis of the left lung in children with congenital heart disease. $\mathcal{F}$ Thorac Cardiovasc Surg lung in children

4 Milne EN. Chest radiology in the surgical patient. Surg Clin North Am 1980;60:1503-18.

(Accepted 10 April 1989) York District Hospital. The samples were divided into two groups according to whether or not the request form indicated that the patient had recently travelled abroad. Few children or elderly patients were among those returning from abroad, and therefore we excluded patients under 15 and over 64. Patients and contacts known to have enteric pathogens in their stools were also excluded.

Stool specimens were examined for salmonella, shigella, and campylobacter and for evidence of intestinal parasites. Specimens from patients returning from Africa, the Middle East, the Indian subcontinent, and the Far East were also examined for vibrios.

Proteeae were detected with modified proteeae isolation medium. ${ }^{3}$ This differed from the original culture medium as it did not contain tyrosine and antibiotics were added to give a final concentration of 
$3 \mathrm{mg}$ vancomycin $/ 1,7 \cdot 5 \mathrm{mg}$ colistin/1, and 12500 units nystatin/l. To recover proteeae present in small numbers both direct and enrichment cultures were used. Gram negative, oxidase negative bacteria growing on proteeae isolation medium and causing characteristic browning of the medium were presumed to be proteeae and were tested for urease activity. No urease positive $P$ alcalifaciens has been reported, so only urease negative isolates were identified further.

When $P$ alcalifaciens was isolated from a stool specimen the predominant lactose fermenting coliform was subcultured from MacConkey's agar and tested for production of heat labile enterotoxin with a coagglutination test. The $\chi^{2}$ test was used to evaluate the significance of the data.

The table summarises the findings. The most obvious difference between the two groups was the increased frequency with which $P$ alcalifaciens was isolated from the patients who had travelled abroad $(\mathrm{p}<0 \cdot 001)$. Most isolates (23 of the 25 ) were obtained from patients returning from Mediterranean countries, but most of the patients who had travelled abroad (194 of the 254) had visited these countries, and no significant association could be shown. None of the patients from whom $P$ alcalifaciens was isolated had travelled together, and

Numbers of isolates of enteric pathogens and proteeae from patients with diarrhoea

\begin{tabular}{lcc}
\hline Organism & $\begin{array}{c}\text { Patients who had } \\
\text { travelled abroad } \\
(\mathbf{n}=254)\end{array}$ & $\begin{array}{c}\text { Patients who had not } \\
\text { travelled abroad } \\
(\mathbf{n}=373)\end{array}$ \\
\hline Salmonella & 22 & $30(\mathrm{p}>0 \cdot 70)$ \\
Campylobacter & 8 & $35(\mathrm{p}<0 \cdot 01)$ \\
Shigella & 6 & Not examined \\
Vibrio & 3 & 3 \\
Giardia lamblia & 1 & 2 \\
Cryptosporidium & 25 & $5(\mathrm{p}<0 \cdot 001)$ \\
Providencia alcalifaciens & 1 & 2 \\
Providencia rustigianii & 82 & $77(\mathrm{p}>0 \cdot 01)$ \\
Urease positive protceae & & \\
\hline
\end{tabular}

none of them were found to carry Escherichia coli that produced heat labile enterotoxin. Urease positive proteeae were isolated with relatively high frequency in both groups but again were significantly more common in the patients who had travelled abroad $(\mathrm{p}<0 \cdot 01)$. Conversely, campylobacter was isolated significantly less commonly in this group $(\mathrm{p}<0 \cdot 01)$.

\section{Comment}

Various factors may have contributed to the differences between the two groups. Patients with travellers' diarrhoea may present for diagnosis later than other patients with diarrhoea, thus reducing the likelihood of the causative agent being isolated. This could account for the lower frequency of isolation of campylobacter in the patients who had travelled abroad. These patients may also be more exposed to faecal bacteria in food and water, resulting in increased carriage of some non-pathogenic bacteria including the proteeae. This seems unlikely to explain the sevenfold or eightfold difference in the distribution of $P$ alcalifaciens between the two groups, but to be certain we would need to examine the normal stools of people who have and have not travelled abroad, and such specimens are not readily available. In the light of previous studies we suggest that $P$ alcalifaciens may be a major cause of diarrhoea among British travellers abroad.

1 Brown GW. Anaerogenic paracolon bacilli associated with gastro-enteritis in children. Med f A A ust 1952;ii:658-64.

2 Ridge LEL, Thomas MEM. Infection with the Providence type of paracolon bacillus in a residential nursery. F Pathol Bacteriol 1955;69:335-7.

3 Sen R. Isolation of strains of the Providence group from cases with diarrhoea in Ibadan, Nigeria, west Africa. Indian f Med Res 1962;50:622-6.

4 Hawkey PM, McCormick A, Simpson RA. Selective and differential medium for the primary isolation of members of the Proteeac. 7 Clin Microbio 1986;23:600-3.

5 Hawkey PM, Potten MR, Stephens M. The use of pre-enrichment for the isolation of small numbers of gentamicin resistant Providencia stuartii from faeces. F Hosp Infect 1982;3:369-74.

(Accepted 17 April 1989)

\section{Gastro-oesophageal disorders in adults with severe mental impairment}

\author{
Joseph Kuruvilla, Peter N Trewby
}

Department of Medicine,

Memorial Hospital,

Darlington, County

Durham DL3 6HX

Joseph Kuruvilla, MB, senior house officer

Peter N Trewby, FRCP, consultant physician

Correspondence to: $\mathrm{Dr}$ Trewby.

$\operatorname{Br}$ Med $\mathcal{J} 1989 ; 299: 95-6$
The prevalence of vomiting in children with severe mental impairment is $10-15 \%$; in three quarters of cases the vomiting is associated with gastro-oesophageal reflux, oesphagitis, or hiatus hernia. ${ }^{12}$ We had gained the impression that vomiting was equally common in mentally impaired adults and so assessed the prevalence of vomiting and other gastrointestinal symptoms and their causes and complications in such patients.

\section{Patients, methods, and results}

We studied 380 long term resident patients in a hospital for adults with severe mental impairment and identified those with recurrent vomiting. We also noted the results of investigations on any of these patients referred to the local district general hospital during 1986-8 because of vomiting and other upper gastrointestinal symptoms.

Thirty nine of the patients ( 26 men and 13 women; mean age 44 (range 21 to 80 ) had recurrent vomiting or other upper gastrointestinal symptoms. Twenty two of them were referred to the district general hospital for investigations: 15 had an upper gastrointestinal endoscopy, four had a barium meal examination, and three needed emergency laparotomies. The main reasons for referral included intractable vomiting and weight loss (13 patients), vomiting and anaemia (11), haematemesis or malaena (four), and dysphagia (one).

Gastro-oesophageal reflux and oesophagitis, with or without a hiatus hernia, was the main cause of vomiting in half of those investigated (table). Of the three patients who needed emergency surgery, one had a haematemesis from a large oesophageal ulcer and required postoperative balloon tamponade, one had an intrathoracic gastric volvulus, and one had a caecal volvulus. In six patients we found either no abnormality to explain the recurrent vomiting or only minor changes such as gastritis, duodenitis, or hiatus hernia without oesphagitis; in these patients the symptoms seemed to be behavioural in origin. The 17 patients with recurrent vomiting who were not investigated all responded to treatment with $\mathrm{H}_{2}$ blockers, metoclopramide, or antacids, which suggested that upper gastrointestinal disease was the cause of their vomiting.

Gastro-oesophageal disorders in severely mentally impaired adults

\begin{tabular}{lc}
\hline Diagnosis & No \\
\hline Hiatus hernia with free reflux & 3 \\
Severe oesophagitis with hiatus hernia & 4 \\
Severe oesophagitis without hiatus hernia & 4 \\
Horizontal volvulus of stomach & 1 \\
Volvulus of caecum & 1 \\
Carcinoma of colon infiltrating stomach & 1 \\
Gastric ulcer & 2 \\
Gastritis/duodenitis & 2 \\
Hiatus hernia & 1 \\
No abnormality & 3 \\
\hline Total & 22 \\
\hline
\end{tabular}

